\title{
A Novel Scoring System for Diagnosing Acute Mesenteric Ischemia in the Emergency Ward: Methodological Issues
}

\author{
Saeid Safiri ${ }^{1,2} \cdot$ Erfan Ayubi $^{3}$
}

Published online: 14 September 2017

(C) Société Internationale de Chirurgie 2017

\section{Dear Editor-in-Chief,}

We were interested to read the article authored by Wang and colleagues that was published in the World Journal of Surgery in 2017 [1]. The authors aimed to develop a scoring system based on traditional physical examinations and serum markers to improve the diagnostic accuracy of acute mesenteric ischemia (AMI). They found that AMI had significantly increased level of white blood cell (WBC) [odds ratio $(\mathrm{OR})=16.11$; 95\% confidence interval (CI): 1.10-235.34], red cell distribution width (RDW; OR $=27.65 ; 95 \%$ CI: $1.53-501.02$ ), mean platelet volume (MPV; OR =16.06; 95\% CI: 1.48-174.50), and D-dimer (OR $=42.91$; 95\% CI: 2.56-718.09), compared with other acute abdomen. Also, they concluded that this scoring system based on easily available parameters can be a useful tool for differentiating AMI from other acute abdomen which needs to be validated in other prospective studies [1]. Although the valuable study has been conducted, some methodological issues need to be taken into account.

First, the authors indicated that their prediction model should be validated in another prospective study, whereas the model can be validated internally through using bootstrapping method which reflects the process of iterative sampling from the study population $[2,3]$.

Erfan Ayubi

aubi65@gmail.com

1 Managerial Epidemiology Research Center, Department of Public Health, School of Nursing and Midwifery, Maragheh University of Medical Sciences, Maragheh, Iran

2 Department of Epidemiology and Biostatistics, School of Public Health, Tehran University of Medical Sciences, Tehran, Iran

3 Department of Epidemiology, School of Public Health, Shahid Beheshti University of Medical Sciences, Tehran, Iran
Second, Wang et al. constructed multivariable logistic regression model but the model construction steps have not been explained. In the standard model construction strategy, the multicollinearity between the candidate variables must be checked in the first step; then, variables with $P$ value $<0.2$ are imported into the multivariable model. Hence, their results may be biased because of multicollinearity between the candidate variables $[4,5]$.

Finally, huge adjusted ORs with substantially wide CIs have reported for WBC, RDW, MPV, and D-dimer which is marker of sparse data bias. This bias can be decreased efficiently through penalization method [6].

A take-home message for the readers is that the prediction model can be validated internally through advanced statistical methods. Also, the standard model construction strategy should be considered in the studies to avoid probable biased results.

\section{Compliance with ethical standards}

Conflict of interest The authors declare that they have no conflicts of interest.

\section{References}

1. Wang Z, Chen J-Q, J-1 Liu, Tian L (2017) A novel scoring system for diagnosing acute mesenteric ischemia in the emergency ward. World J Surg 2017:1-9. doi:10.1007/s00268-017-3984-9

2. Steyerberg E (2008) Clinical prediction models: a practical approach to development, validation, and updating. Springer, Berlin

3. Ayubi E, Safiri S (2017) Comments on CHA2DS2-VASc score in the prediction of ischemic bowel disease among patients with atrial fibrillation. Int J Cardiol 242:9

4. Jewell NP (2003) Statistics for epidemiology. CRC Press, Boca Raton

5. Hosmer DW Jr, Lemeshow S, Sturdivant RX (2013) Applied logistic regression. Wiley, Hoboken

6. Greenland S, Mansournia MA, Altman DG (2016) Sparse data bias: a problem hiding in plain sight. bmj 352:i1981 